\title{
Control of DFIG-Based Wind Generation Systems under Unbalanced Network Supply
}

\author{
Yi Wang, and Lie $\mathrm{Xu}$ \\ School of Electronics, Electrical Engineering \& Computer Science \\ Queen's University of Belfast \\ Stranmillis Road, Belfast, BT9 5AH, United Kingdom
}

\begin{abstract}
This paper develops a dynamic model and control scheme for DFIG systems to improve the performance and stability under unbalanced grid conditions. A dynamic DFIG model containing the positive and negative sequence components is presented using stator voltage orientation. The proposed model accurately illustrates the active power, reactive power and torque oscillations, and provides a basis for DFIG control system design during unbalanced network supply. Various control targets such as eliminating the oscillations of the torque, active/reactive power are discussed and the required rotor negative sequence current for fulfilling different control targets are described. Performance of a DFIG-based wind turbine under unbalanced condition using the proposed control method is evaluated by simulation studies using Matlab/Simulink. The proposed control scheme significantly attenuates the DFIG torque or active power oscillations during network unbalance whereas significant torque/power oscillations exist with the conventional control schemes.
\end{abstract}

\section{INTRODUCTION}

Nowadays, doubly-fed induction generators (DFIGs) are commonly used for large wind turbines operating at variable speed. For maintaining continuous operation of such wind turbines during power system disturbances, i.e., fault ride through capability, extensive studies have been carried out in the last few years. However, symmetrical voltages are considered in most cases [1-4]. In reality, asymmetric faults occur more frequently than symmetric faults in transmission system. The stator current of a DFIG could be highly unbalanced even with a small unbalanced grid voltage if no unbalance control were considered. The unbalanced voltage and current cause a number of problems such as overheating of stator windings, extra mechanical stresses due to torque pulsation, and output power pulsations.

Control and operation of DFIG systems during network unbalance were studied in [5-8]. However, in [5, 6], the focus was only on controlling the grid side converter to provide similar functions as a STATCOM. In [7,8], control of DFIG for compensating torque pulsation under unbalanced supply voltage was investigated. However, since the required rotor compensating current oscillates at twice the line frequency, the controller needs to be carefully tuned to provide the required system response at double supply frequency. Furthermore, the impact of unbalanced stator voltage on the stator and rotor currents has not been fully defined and the relationships between the pulsations of the torque, the stator active/reactive power, and the rotor currents have not been fully established.
The aim of this paper is to develop a dynamic model and control scheme for DFIG systems to improve the performance and stability under unbalanced operation. A dynamic model of DFIG systems based on stator voltage orientation (SVO) under unbalanced condition is presented. The relationships between torque, output power, and unbalanced stator voltage supply and rotor current are fully established. Various control targets, such as no torque pulsation, or no power oscillation, are discussed. Based on the developed model, system control using two separate controllers, i.e., positive sequence controller and negative sequence controller are designed. The performance of a DFIG system operating under unbalanced conditions using the proposed control strategy is evaluated by simulations using Matlab/Simulink.

\section{DFIG MODELING UNDER UNBALANCED CONDITIONS}

The existing DFIG models are primarily developed on the basis of symmetric grid voltage. For unbalanced supply, both positive and negative sequence components of voltage and current need to be considered in order to accurately describe the system behavior. In order to obtain a decoupled control between torque/active power and reactive power, $\mathrm{d}$-axis in the synchronous reference frame is generally oriented along the stator flux vector or the stator voltage vector. In this paper, stator voltage orientation is used.

A complex vector equivalent circuit of a DFIG in an arbitrary dq reference frame rotating at angular speed of $\omega_{\mathrm{e}}$ is shown in Fig. 1 [9]. The flux and voltage equations in the synchronous reference frame are given as

$$
\begin{aligned}
& \boldsymbol{\psi}_{\mathrm{s}}=L_{\mathrm{s}} \boldsymbol{I}_{\mathrm{s}}+L_{\mathrm{m}} \boldsymbol{I}_{\mathrm{r}} \\
& \boldsymbol{\psi}_{\mathrm{r}}=L_{\mathrm{r}} \boldsymbol{I}_{\mathrm{r}}+L_{\mathrm{m}} \boldsymbol{I}_{\mathrm{s}} \\
& \boldsymbol{V}_{\mathrm{s}}=R_{\mathrm{s}} \boldsymbol{I}_{\mathrm{s}}+\frac{\mathrm{d} \boldsymbol{\psi}_{\mathrm{s}}}{\mathrm{d} t}+\mathrm{j} \omega_{\mathrm{e}} \boldsymbol{\psi}_{\mathrm{s}} \\
& \boldsymbol{V}_{\mathrm{r}}=R_{\mathrm{r}} \boldsymbol{I}_{\mathrm{r}}+\frac{\mathrm{d} \boldsymbol{\psi}_{\mathrm{r}}}{\mathrm{d} t}+\mathrm{j}\left(\omega_{\mathrm{e}}-\omega_{\mathrm{r}}\right) \boldsymbol{\psi}_{\mathrm{r}}
\end{aligned}
$$

where $\psi, V$ and $I$ represent the flux, voltage and current. Subscripts $\mathrm{s}$ and $\mathrm{r}$ denote the stator and rotor quantities respectively. $L_{\mathrm{s}}$ and $L_{\mathrm{r}}$ are the stator and rotor self inductances, $L_{\mathrm{m}}$ is the mutual inductance, and $\omega_{\mathrm{r}}$ is the rotor angular frequency.

According to (1), the rotor flux, stator current and rotor voltage can be given as 


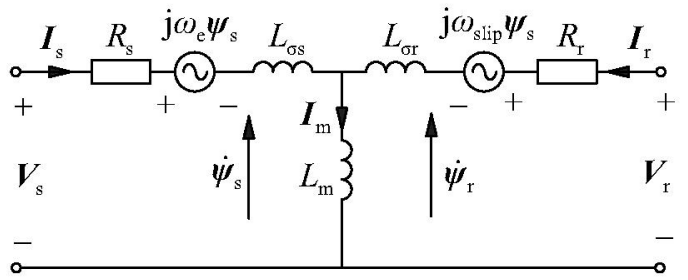

Fig. 1. The complex vector equivalent circuit of a DFIG in synchronous reference frame

$$
\begin{aligned}
\boldsymbol{\psi}_{\mathrm{r}}= & \frac{L_{\mathrm{m}}}{L_{\mathrm{s}}} \boldsymbol{\psi}_{\mathrm{s}}+\sigma L_{\mathrm{r}} \boldsymbol{I}_{\mathrm{r}} \\
\boldsymbol{I}_{\mathrm{s}}= & \frac{1}{L_{\mathrm{s}}}\left(\boldsymbol{\psi}_{\mathrm{s}}-L_{\mathrm{m}} \boldsymbol{I}_{\mathrm{r}}\right) \\
\boldsymbol{V}_{\mathrm{r}}= & R_{\mathrm{r}} \boldsymbol{I}_{\mathrm{r}}+\sigma L_{\mathrm{r}} \frac{\mathrm{d} \boldsymbol{I}_{\mathrm{r}}}{\mathrm{d} t}+\frac{L_{\mathrm{m}}}{L_{\mathrm{s}}} \frac{\mathrm{d} \boldsymbol{\psi}_{\mathrm{s}}}{\mathrm{d} t} \\
& +\mathrm{j} \omega_{\text {slip }}\left(\sigma L_{\mathrm{r}} \boldsymbol{I}_{\mathrm{r}}+\frac{L_{\mathrm{m}}}{L_{\mathrm{s}}} \boldsymbol{\psi}_{\mathrm{s}}\right)
\end{aligned}
$$

where $\sigma=1-L_{\mathrm{m}}^{2} / L_{\mathrm{r}} L_{\mathrm{s}}$ is the leakage factor, and $\omega_{\text {slip }}=\omega_{\mathrm{e}}-\omega_{\mathrm{r}}$ is the slip frequency.

Assuming no zero sequence components, the vector $\boldsymbol{F}$ which represents three-phase quantities such as voltage, current and flux can be decomposed into positive and negative sequence components when the network is unbalanced, as [10]

$$
\begin{aligned}
\boldsymbol{F}(t) & =F^{\mathrm{p}} e^{\mathrm{j}\left(\omega_{\mathrm{e}} t+\varphi^{\mathrm{p}}\right)}+F^{\mathrm{n}} e^{\mathrm{j}\left(-\omega_{\mathrm{e}} t+\varphi^{\mathrm{n}}\right)} \\
& =\left(F_{\mathrm{d}}^{\mathrm{p}}+\mathrm{j} F_{\mathrm{q}}^{\mathrm{p}}\right) e^{\mathrm{j} \omega_{\mathrm{e}} t}+\left(F_{\mathrm{d}}^{\mathrm{n}}+\mathrm{j} F_{\mathrm{q}}^{\mathrm{n}}\right) e^{-\mathrm{j} \omega_{\mathrm{e}} t}
\end{aligned}
$$

where superscripts $\mathrm{p}$ and $\mathrm{n}$ denote the positive and negative components respectively, whereas subscripts $d$ and $q$ denote the $\mathrm{d}$-axis and $\mathrm{q}$-axis components respectively.

The positive and negative sequence components in the stationary reference frame are then transformed into $(\mathrm{dq})+$ and $(\mathrm{dq})$ - reference frames rotating at angular speeds of $\omega_{\mathrm{e}}$ and $-\omega_{\mathrm{e}}$ respectively. The spatial relationship of these three reference frames is shown in Fig. 2, where $\mathrm{d}+$ axis is oriented to the positive sequence stator voltage vector.

For a DFIG, its instantaneous stator power can be expressed in the $(\mathrm{dq})+$ reference frame as

$$
\begin{aligned}
p_{\mathrm{s}}+\mathrm{j} q_{\mathrm{s}} & =-\frac{3}{2} \boldsymbol{V}_{\mathrm{s}}(t) \boldsymbol{I}_{\mathrm{s}}^{*}(t) \\
& =-\frac{3}{2 L_{\mathrm{s}}} \boldsymbol{V}_{\mathrm{s}}(t)\left(\psi_{\mathrm{s}}^{*}(t)-L_{\mathrm{m}} \boldsymbol{I}_{\mathrm{r}}^{*}(t)\right)
\end{aligned}
$$

According to (3), the stator voltage $V_{s}$, stator flux $\psi_{\mathrm{s}}$ and rotor current $\boldsymbol{I}_{\mathrm{r}}$ can be expressed using their respective positive and negative sequence components. Thus, based on (4), the active and reactive power can then be expressed as

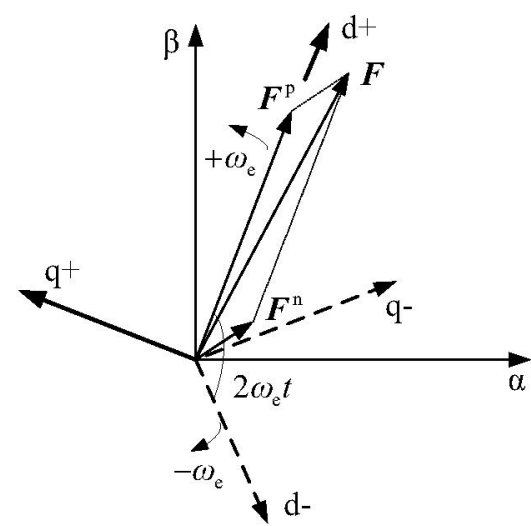

Fig. 2 Positive and negative sequence components and relationship between the different reference frames

$$
\begin{aligned}
& p_{\mathrm{s}}=P_{\mathrm{s} \_\mathrm{av}}+P_{\mathrm{s}_{-} \sin 2} \sin \left(2 \omega_{\mathrm{e}} t\right)+P_{s_{-} \cos 2} \cos \left(2 \omega_{\mathrm{e}} t\right) \\
& q_{\mathrm{s}}=Q_{\mathrm{s}_{-} \mathrm{av}}+Q_{\mathrm{s}_{-} \sin 2} \sin \left(2 \omega_{\mathrm{e}} t\right)+Q_{\mathrm{s}_{-} \cos 2} \cos \left(2 \omega_{\mathrm{e}} t\right)
\end{aligned}
$$

where $P_{\mathrm{s}}, Q_{\mathrm{s}}, P_{\mathrm{s}_{-} \sin 2}, P_{\mathrm{s}_{-} \cos 2}, Q_{\mathrm{s}_{-} \sin 2}$ and $Q_{\mathrm{s}_{-} \cos 2}$ are given by

$$
\left[\begin{array}{c}
P_{\mathrm{s}_{-} \mathrm{av}} \\
Q_{\mathrm{s}_{-} \mathrm{av}} \\
P_{\mathrm{s}_{-} \sin 2} \\
P_{\mathrm{s}_{-} \cos 2} \\
Q_{\mathrm{s}_{-} \sin 2} \\
Q_{\mathrm{s}_{-} \cos 2}
\end{array}\right]=-\frac{3}{2 L_{\mathrm{s}}}\left[\begin{array}{cccc}
V_{\mathrm{sd}}^{\mathrm{p}} & V_{\mathrm{sq}}^{\mathrm{p}} & V_{\mathrm{sd}}^{\mathrm{n}} & V_{\mathrm{sq}}^{\mathrm{n}} \\
V_{\mathrm{sq}}^{\mathrm{p}} & -V_{\mathrm{sd}}^{\mathrm{p}} & V_{\mathrm{sq}}^{\mathrm{n}} & -V_{\mathrm{sd}}^{\mathrm{n}} \\
V_{\mathrm{sq}}^{\mathrm{n}} & -V_{\mathrm{sd}}^{\mathrm{n}} & -V_{\mathrm{sq}}^{\mathrm{p}} & V_{\mathrm{sd}}^{\mathrm{p}} \\
V_{\mathrm{sd}}^{\mathrm{n}} & V_{\mathrm{sq}}^{\mathrm{n}} & V_{\mathrm{sd}}^{\mathrm{p}} & V_{\mathrm{sq}}^{\mathrm{p}} \\
-V_{\mathrm{sd}}^{\mathrm{n}} & -V_{\mathrm{sq}}^{\mathrm{n}} & V_{\mathrm{sd}}^{\mathrm{p}} & V_{\mathrm{sq}}^{\mathrm{p}} \\
V_{\mathrm{sq}}^{\mathrm{n}} & -V_{\mathrm{sd}}^{\mathrm{n}} & V_{\mathrm{sq}}^{\mathrm{p}} & -V_{\mathrm{sd}}^{\mathrm{p}}
\end{array}\right]\left(\left[\begin{array}{c}
\psi_{\mathrm{sd}}^{\mathrm{p}} \\
\psi_{\mathrm{sq}}^{\mathrm{p}} \\
\psi_{\mathrm{sd}}^{\mathrm{n}} \\
\psi_{\mathrm{sq}}^{\mathrm{n}}
\end{array}\right]-L_{\mathrm{m}}\left[\begin{array}{c}
I_{\mathrm{rd}}^{\mathrm{p}} \\
I_{\mathrm{rq}}^{\mathrm{p}} \\
I_{\mathrm{rd}}^{\mathrm{n}} \\
I_{\mathrm{rq}}^{\mathrm{n}}
\end{array}\right]\right)
$$

The electromagnetic torque $T_{\mathrm{e}}$ is given by

$$
\begin{aligned}
T_{\mathrm{e}} & =\frac{3}{2} p_{\mathrm{n}} \operatorname{Im}\left[\psi_{\mathrm{s}}(t) \boldsymbol{I}_{\mathrm{s}}^{*}(t)\right] \\
& =\frac{3}{2} p_{\mathrm{n}} \operatorname{Im}\left[\frac{1}{L_{s}} \psi_{\mathrm{s}}(t)\left(\psi_{\mathrm{s}}^{*}(t)-L_{\mathrm{m}} \boldsymbol{I}_{\mathrm{r}}^{*}(t)\right)\right] \\
& =-\frac{3 L_{\mathrm{m}}}{2 L_{s}} p_{\mathrm{n}} \operatorname{Im}\left[\psi_{\mathrm{s}}(t) \boldsymbol{I}_{\mathrm{r}}^{*}(t)\right] \\
& =T_{\mathrm{e} \_a v}+T_{\mathrm{e}_{-} \sin 2} \sin \left(2 \omega_{\mathrm{e}} t\right)+T_{\mathrm{e}_{-} \cos 2} \cos \left(2 \omega_{\mathrm{e}} t\right)
\end{aligned}
$$

where $T_{\mathrm{e}_{-} \text {av }}, T_{\mathrm{e}_{-} \sin 2}$ and $T_{\mathrm{e}_{-} \cos 2}$ are given by

$$
\left[\begin{array}{c}
T_{\mathrm{e} \_\mathrm{a}} \\
T_{\mathrm{e} \_\sin 2} \\
T_{\mathrm{e} \_\cos 2}
\end{array}\right]=-\frac{3 p_{\mathrm{n}} L_{\mathrm{m}}}{2 L_{\mathrm{s}}}\left[\begin{array}{cccc}
\psi_{\mathrm{sq}}^{\mathrm{p}} & -\psi_{\mathrm{sd}}^{\mathrm{p}} & \psi_{\mathrm{sq}}^{\mathrm{n}} & -\psi_{\mathrm{sd}}^{\mathrm{n}} \\
-\psi_{\mathrm{sd}}^{\mathrm{n}} & -\psi_{\mathrm{sq}}^{\mathrm{n}} & \psi_{\mathrm{sd}}^{\mathrm{p}} & \psi_{\mathrm{sq}}^{\mathrm{p}} \\
\psi_{\mathrm{sq}}^{\mathrm{n}} & -\psi_{\mathrm{sd}}^{\mathrm{n}} & \psi_{\mathrm{sq}}^{\mathrm{p}} & -\psi_{\mathrm{sd}}^{\mathrm{p}}
\end{array}\right]\left[\begin{array}{c}
I_{\mathrm{rd}}^{\mathrm{p}} \\
I_{\mathrm{rq}}^{\mathrm{p}} \\
I_{\mathrm{rd}}^{\mathrm{n}} \\
I_{\mathrm{rq}}^{\mathrm{n}}
\end{array}\right] .
$$

According to (1) and neglecting the stator resistance, in the $(\mathrm{dq})+$ reference frame, the stator voltage is given by 


$$
\begin{aligned}
\boldsymbol{V}_{\mathrm{s}}(t) & =\frac{\mathrm{d}\left(\boldsymbol{\psi}_{\mathrm{s}}^{\mathrm{p}}+\boldsymbol{\psi}_{\mathrm{s}}^{\mathrm{n}} e^{-\mathrm{j} 2 \omega_{\mathrm{e}} t}\right)}{\mathrm{d} t}+\mathrm{j} \omega_{\mathrm{e}}\left(\boldsymbol{\psi}_{\mathrm{s}}^{\mathrm{p}}+\boldsymbol{\psi}_{\mathrm{s}}^{\mathrm{n}} e^{-\mathrm{j} 2 \omega_{\mathrm{e}} t}\right) \\
& =\mathrm{j} \omega_{\mathrm{e}} \boldsymbol{\psi}_{\mathrm{s}}^{\mathrm{p}}-\mathrm{j} \omega_{\mathrm{e}} \boldsymbol{\psi}_{\mathrm{s}}^{\mathrm{n}} e^{-\mathrm{j} 2 \omega_{\mathrm{e}} t} \\
& =\boldsymbol{V}_{\mathrm{s}}^{\mathrm{p}}+\boldsymbol{V}_{\mathrm{s}}^{\mathrm{n}} e^{-\mathrm{j} 2 \omega_{\mathrm{e}} t}
\end{aligned}
$$

Thus the relationship between stator voltage and stator flux is given as

$$
\begin{aligned}
& \boldsymbol{V}_{\mathrm{s}}^{\mathrm{p}}=\mathrm{j} \omega_{\mathrm{e}} \boldsymbol{\psi}_{\mathrm{s}}^{\mathrm{p}} \\
& \boldsymbol{V}_{\mathrm{s}}^{\mathrm{n}}=-\mathrm{j} \omega_{\mathrm{e}} \boldsymbol{\psi}_{\mathrm{s}}^{\mathrm{n}}
\end{aligned}
$$

Due to the fact that the $d+$ axis is oriented to the positive sequence stator voltage vector, its $\mathrm{q}^{+}$axis component $V_{\mathrm{sq}}^{\mathrm{p}}$ can be regarded as zero. Substituting (10) into (6) and (8) yields, then

$$
\begin{aligned}
& {\left[\begin{array}{c}
P_{\mathrm{s}-\mathrm{av}} \\
Q_{\mathrm{s}_{-} \mathrm{av}} \\
P_{\mathrm{s}_{-} \sin 2} \\
P_{\mathrm{s}_{-} \cos 2} \\
Q_{\mathrm{s}_{-} \sin 2} \\
Q_{\mathrm{s}_{-} \cos 2}
\end{array}\right]=-\frac{3}{2 L_{\mathrm{s}} \omega_{\mathrm{e}}}\left[\begin{array}{c}
0 \\
\left(V_{\mathrm{s}}^{\mathrm{p}}\right)^{2}-\left(V_{\mathrm{s}}^{\mathrm{n}}\right)^{2} \\
2 V_{\mathrm{sd}}^{\mathrm{p}} V_{\mathrm{sd}}^{\mathrm{n}} \\
-2 V_{\mathrm{sd}}^{\mathrm{p}} V_{\mathrm{sq}}^{\mathrm{n}} \\
0 \\
0
\end{array}\right]+} \\
& \frac{3 L_{\mathrm{m}}}{2 L_{\mathrm{s}}}\left[\begin{array}{cccc}
V_{\mathrm{sd}}^{\mathrm{p}} & 0 & V_{\mathrm{sd}}^{\mathrm{n}} & V_{\mathrm{sq}}^{\mathrm{n}} \\
0 & -V_{\mathrm{sd}}^{\mathrm{p}} & V_{\mathrm{sq}}^{\mathrm{n}} & -V_{\mathrm{sd}}^{\mathrm{n}} \\
V_{\mathrm{sq}}^{\mathrm{n}} & -V_{\mathrm{sd}}^{\mathrm{n}} & 0 & V_{\mathrm{sd}}^{\mathrm{p}} \\
V_{\mathrm{sd}}^{\mathrm{n}} & V_{\mathrm{sq}}^{\mathrm{n}} & V_{\mathrm{sd}}^{\mathrm{p}} & 0 \\
-V_{\mathrm{sd}}^{\mathrm{n}} & -V_{\mathrm{sq}}^{\mathrm{n}} & V_{\mathrm{sd}}^{\mathrm{p}} & 0 \\
V_{\mathrm{sq}}^{\mathrm{n}} & -V_{\mathrm{sd}}^{\mathrm{n}} & 0 & -V_{\mathrm{sd}}^{\mathrm{p}}
\end{array}\right]\left[\begin{array}{c}
I_{\mathrm{rd}}^{\mathrm{p}} \\
I_{\mathrm{rq}}^{\mathrm{p}} \\
I_{\mathrm{rd}}^{\mathrm{n}} \\
I_{\mathrm{rq}}^{\mathrm{n}}
\end{array}\right] \\
& {\left[\begin{array}{c}
T_{\mathrm{e} \_ \text {av }} \\
T_{\mathrm{e} \_\sin 2} \\
T_{\mathrm{e} \_ \text {cos } 2}
\end{array}\right]=-\frac{3 p_{\mathrm{n}} L_{\mathrm{m}}}{2 \omega_{\mathrm{e}} L_{\mathrm{s}}}\left[\begin{array}{cccc}
-V_{\mathrm{sd}}^{\mathrm{p}} & 0 & V_{\mathrm{sd}}^{\mathrm{n}} & V_{\mathrm{sq}}^{\mathrm{n}} \\
V_{\mathrm{sq}}^{\mathrm{n}} & -V_{\mathrm{sd}}^{\mathrm{n}} & 0 & -V_{\mathrm{sd}}^{\mathrm{p}} \\
V_{\mathrm{sd}}^{\mathrm{n}} & V_{\mathrm{sq}}^{\mathrm{n}} & -V_{\mathrm{sd}}^{\mathrm{p}} & 0
\end{array}\right]\left[\begin{array}{c}
I_{\mathrm{rd}}^{\mathrm{p}} \\
I_{\mathrm{rq}}^{\mathrm{p}} \\
I_{\mathrm{rd}}^{\mathrm{n}} \\
I_{\mathrm{rq}}^{\mathrm{n}}
\end{array}\right] .}
\end{aligned}
$$

According to (11) and (12), the following conclusions can be drawn:

(1) To minimize the stator active power fluctuation, i.e., $P_{\mathrm{s}_{-} \sin 2}=0$ and $P_{\mathrm{s}_{-} \cos 2}=0$, according to $(11)$ the negative sequence rotor current needs to be controlled as

$$
\begin{aligned}
& I_{\mathrm{rd}}^{\mathrm{n}}=-\frac{1}{V_{\mathrm{s}}^{\mathrm{p}}}\left(V_{\mathrm{sd}}^{\mathrm{n}} I_{\mathrm{rd}}^{\mathrm{p}}+V_{\mathrm{sq}}^{\mathrm{n}} I_{\mathrm{rq}}^{\mathrm{p}}\right)-\frac{2 V_{\mathrm{sq}}^{\mathrm{n}}}{\omega_{\mathrm{e}} L_{\mathrm{m}}} \\
& I_{\mathrm{rq}}^{\mathrm{n}}=\frac{1}{V_{\mathrm{s}}^{\mathrm{p}}}\left(V_{\mathrm{sd}}^{\mathrm{n}} I_{\mathrm{rq}}^{\mathrm{p}}-V_{\mathrm{sq}}^{\mathrm{n}} I_{\mathrm{rd}}^{\mathrm{p}}\right)+\frac{2 V_{\mathrm{sd}}^{\mathrm{n}}}{\omega_{\mathrm{e}} L_{\mathrm{m}}}
\end{aligned}
$$

Under such condition, the average stator active and reactive power and the electromagnetic torque are given by

$$
\begin{aligned}
& P_{\mathrm{s} \_\mathrm{av}}=\frac{3 L_{\mathrm{m}}}{2 L_{\mathrm{s}}} \frac{\left(V_{\mathrm{s}}^{\mathrm{p}}\right)^{2}-\left(V_{\mathrm{s}}^{\mathrm{n}}\right)^{2}}{V_{\mathrm{s}}^{\mathrm{p}}} I_{\mathrm{rd}}^{\mathrm{p}} \\
& Q_{\mathrm{s} \_\mathrm{av}}=-\frac{3}{2 L_{\mathrm{s}}} \frac{\left(V_{\mathrm{s}}^{\mathrm{p}}\right)^{2}+\left(V_{\mathrm{s}}^{\mathrm{n}}\right)^{2}}{V_{\mathrm{s}}^{\mathrm{p}}}\left(\frac{V_{\mathrm{s}}^{\mathrm{p}}}{\omega_{\mathrm{e}}}+L_{\mathrm{m}} I_{\mathrm{rq}}^{\mathrm{p}}\right) . \\
& T_{\mathrm{e} \_\mathrm{av}}=\frac{3 p_{\mathrm{n}} L_{\mathrm{m}}}{2 \omega_{\mathrm{e}} L_{\mathrm{s}}} \frac{\left(V_{\mathrm{s}}^{\mathrm{p}}\right)^{2}+\left(V_{\mathrm{s}}^{\mathrm{n}}\right)^{2}}{V_{\mathrm{s}}^{\mathrm{p}}} I_{\mathrm{rd}}^{\mathrm{p}}
\end{aligned}
$$

(2) To remove the oscillations of electromagnetic torque, i.e., $T_{\mathrm{e}_{-} \sin 2}=0$ and $T_{\mathrm{e}_{-} \cos 2}=0$, according to (12) the required negative sequence rotor current is given as

$$
\begin{aligned}
& I_{\mathrm{rd}}^{\mathrm{n}}=\frac{1}{V_{\mathrm{s}}^{\mathrm{p}}}\left(V_{\mathrm{sd}}^{\mathrm{n}} I_{\mathrm{rd}}^{\mathrm{p}}+V_{\mathrm{sq}}^{\mathrm{n}} I_{\mathrm{rq}}^{\mathrm{p}}\right) \\
& I_{\mathrm{rq}}^{\mathrm{n}}=\frac{1}{V_{\mathrm{s}}^{\mathrm{p}}}\left(V_{\mathrm{sq}}^{\mathrm{n}} I_{\mathrm{rd}}^{\mathrm{p}}-V_{\mathrm{sd}}^{\mathrm{n}} I_{\mathrm{rq}}^{\mathrm{p}}\right)
\end{aligned} .
$$

Thus the average stator active and reactive power and the electromagnetic torque are given by

$$
\begin{aligned}
& P_{\mathrm{s} \_\mathrm{av}}=\frac{3 L_{\mathrm{m}}}{2 L_{\mathrm{s}}} \frac{\left(V_{\mathrm{s}}^{\mathrm{p}}\right)^{2}+\left(V_{\mathrm{s}}^{\mathrm{n}}\right)^{2}}{V_{\mathrm{s}}^{\mathrm{p}}} I_{\mathrm{rd}}^{\mathrm{p}} \\
& Q_{\mathrm{s} \_\mathrm{av}}=-\frac{3}{2 L_{\mathrm{s}}} \frac{\left(V_{\mathrm{s}}^{\mathrm{p}}\right)^{2}-\left(V_{\mathrm{s}}^{\mathrm{n}}\right)^{2}}{V_{\mathrm{s}}^{\mathrm{p}}}\left(\frac{V_{\mathrm{s}}^{\mathrm{p}}}{\omega_{\mathrm{e}}}+L_{\mathrm{m}} I_{\mathrm{rq}}^{\mathrm{p}}\right) . \\
& T_{\mathrm{e} \_\mathrm{av}}=\frac{3 p_{\mathrm{n}} L_{\mathrm{m}}}{2 \omega_{\mathrm{e}} L_{\mathrm{s}}} \frac{\left(V_{\mathrm{s}}^{\mathrm{p}}\right)^{2}-\left(V_{\mathrm{s}}^{\mathrm{n}}\right)^{2}}{V_{\mathrm{s}}^{\mathrm{p}}} I_{\mathrm{rd}}^{\mathrm{p}}
\end{aligned}
$$

Equations (14) and (16) indicate that the average stator active power/torque and reactive power can be controlled by regulating $I_{\mathrm{rd}}^{\mathrm{p}}$ and $I_{\mathrm{rq}}^{\mathrm{p}}$ respectively. In the mean while, controlling $I_{\mathrm{rd}}^{\mathrm{n}}$ and $I_{\mathrm{rq}}^{\mathrm{n}}$ can eliminate the oscillations of either stator active power based on (13), or electromagnetic torque based on (15). Therefore, the average values of active power/torque and reactive power can be controlled independently using a positive sequence controller, and their oscillations can be eliminated using a negative sequence controller.

\section{SYSTEM CONTROL}

Fig. 3 shows the schematic diagram of a vector control system to provide precise control of the rotor positive and negative sequence currents

\section{A. Voltage phase-locked loop}

For stator voltage orientation, the stator voltage frequency and angle can be measured by phase-locked loop (PLL). Various software PLL techniques have been proposed to obtain accurate phase information. Under unbalanced conditions, grid voltage contains positive and negative sequence components, and the PLL should lock the positive sequence voltage phase. A band-trap filter turned at twice the grid frequency can be 


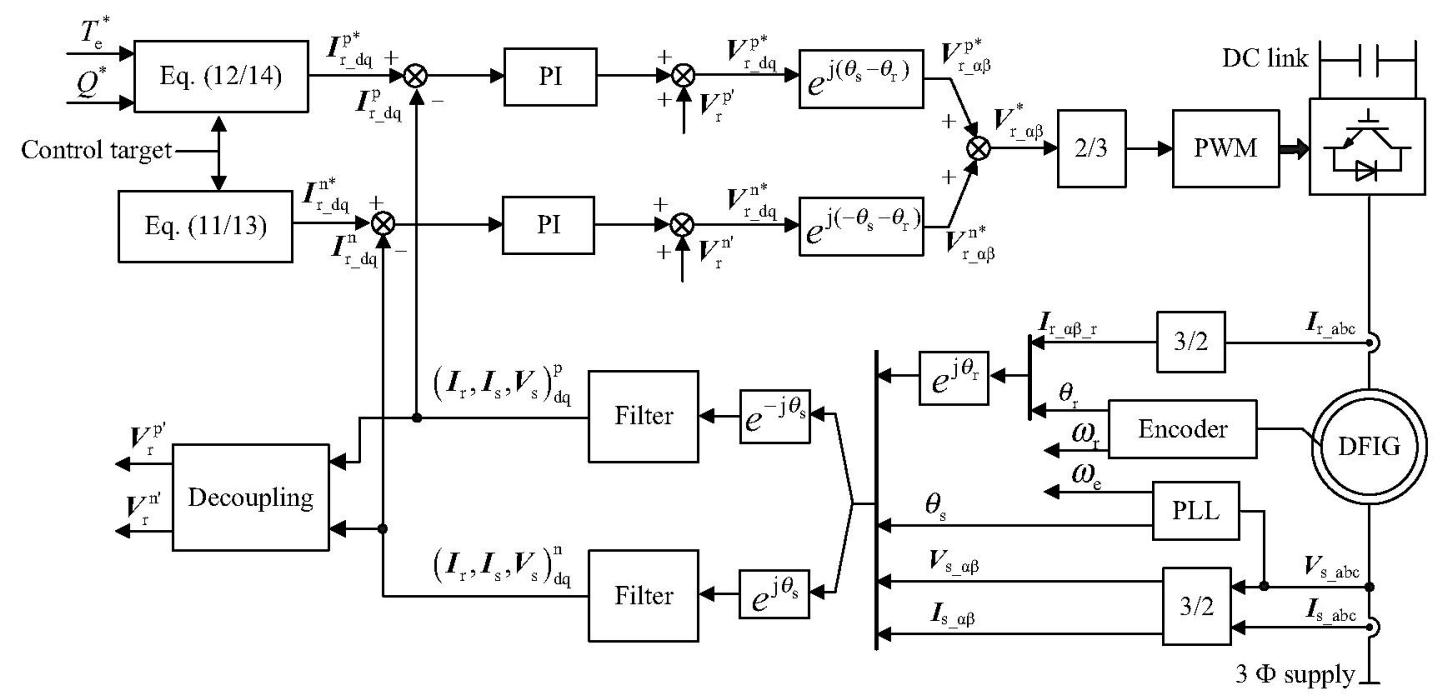

Fig. 3 Schematic diagram of the control system based on the proposed DFIG model

employed to reduce the negative sequence components in PLL [9].

\section{B. Positive and negative sequence components separation}

Once the $(\mathrm{dq})+$ and $(\mathrm{dq})-$ reference frames being determined using the PLL, the various measured quantities can be transformed into the synchronous reference frame. The positive and negative sequence components transformed into $(\mathrm{dq})+$ and (dq)- frames are given by

$$
\begin{aligned}
& \boldsymbol{F}_{\mathrm{dq}+}(t)=\boldsymbol{F}(t) e^{-\mathrm{j} \omega_{\mathrm{g}} t}=\boldsymbol{F}^{\mathrm{p}}+\boldsymbol{F}^{\mathrm{n}} e^{-2 \mathrm{j} \omega_{\mathrm{g}} t} \\
& \boldsymbol{F}_{\mathrm{dq}-}(t)=\boldsymbol{F}(t) e^{\mathrm{j} \omega_{\mathrm{e}} t}=\boldsymbol{F}^{\mathrm{p}} e^{\mathrm{j} 2 \omega_{\mathrm{c}} t}+\boldsymbol{F}^{\mathrm{n}}
\end{aligned} .
$$

Therefore, in $(\mathrm{dq})+$ frame, positive sequence components appear as DC values while negative components oscillate at $2 \omega_{\mathrm{e}}$. The same applies to the transformation in $(\mathrm{dq})^{-}$frame. Thus in order to separate the positive and negative sequence components, the quantities in stationary frame are transformed into $(\mathrm{dq})+$ and $(\mathrm{dq})-$ frames firstly, and then a band-trap filter tuned at $2 \omega_{\mathrm{e}}$ is used to remove the oscillating terms.

\section{Positive and negative sequence controllers}

In the proposed strategy, two control loops, i.e., a positive sequence controller implemented in the $(\mathrm{dq})+$ reference frame, and a negative sequence controller implemented in the (dq)reference frame are used. Under unbalanced conditions, the following control targets can be realized by regulating the rotor negative current at different reference values.

Target 1. Constant stator output active power, i.e. no active power pulsation at twice the grid frequency.

Target 2. Constant electromagnetic torque to reduce the mechanical stress on the turbine system.

For target 1, the reference values of positive and negative controllers can be calculated by (14) and (13) respectively. For target 2 , the reference values of positive and negative controllers can be calculated by (16) and (15) respectively.
Furthermore, the negative current reference values should set an appropriate limitation to ensure the generator windings not overheating.

PI controllers are used for regulating the rotor positive and negative sequence currents to follow their respective varying reference points. The required rotor control voltages in the positive and negative sequence frames are given by

$$
\begin{aligned}
& \boldsymbol{V}_{\mathrm{r}}^{\mathrm{p}^{*}}=k_{\mathrm{p} 1}\left(\boldsymbol{I}_{\mathrm{r}}^{\mathrm{p}^{*}}-\boldsymbol{I}_{\mathrm{r}}^{\mathrm{p}}\right)+k_{\mathrm{i} 1} \int\left(\boldsymbol{I}_{\mathrm{r}}^{\mathrm{p}^{*}}-\boldsymbol{I}_{\mathrm{r}}^{\mathrm{p}}\right) \mathrm{d} t+\boldsymbol{V}_{\mathrm{r}}^{\mathrm{p}^{\prime}} \\
& \boldsymbol{V}_{\mathrm{r}}^{\mathrm{n}^{*}}=k_{\mathrm{p} 2}\left(\boldsymbol{I}_{\mathrm{r}}^{\mathrm{n}^{*}}-\boldsymbol{I}_{\mathrm{r}}^{\mathrm{n}}\right)+k_{\mathrm{i} 2} \int\left(\boldsymbol{I}_{\mathrm{r}}^{\mathrm{n}^{*}}-\boldsymbol{I}_{\mathrm{r}}^{\mathrm{n}}\right) \mathrm{d} t+\boldsymbol{V}_{\mathrm{r}}^{\mathrm{n}^{\prime}}
\end{aligned}
$$

where $\boldsymbol{V}_{\mathrm{r}}^{\mathrm{p}^{\prime}}$ and $\boldsymbol{V}_{\mathrm{r}}^{\mathrm{n}^{\prime}}$ are the decoupling voltages, which can be calculated by

$$
\begin{aligned}
& V_{\mathrm{rd}}^{\mathrm{p}^{\prime}}=-\omega_{\mathrm{slip}} \sigma L_{\mathrm{r}} I_{\mathrm{rq}}^{\mathrm{p}}+\frac{\omega_{\mathrm{slip}} L_{\mathrm{m}}}{\omega_{\mathrm{e}} L_{\mathrm{s}}} V_{\mathrm{sd}}^{\mathrm{p}} \\
& V_{\mathrm{rq}}^{\mathrm{p}^{\prime}}=\omega_{\text {slip }} \sigma L_{\mathrm{r}} I_{\mathrm{rd}}^{\mathrm{p}}+\frac{\omega_{\mathrm{slip}} L_{\mathrm{m}}}{\omega_{\mathrm{e}} L_{\mathrm{s}}} V_{\mathrm{sq}}^{\mathrm{p}} \\
& V_{\mathrm{rd}}^{\mathrm{n}^{\prime}}=-\omega_{\mathrm{slip}} \sigma L_{\mathrm{r}} I_{\mathrm{rq}}^{\mathrm{n}}-\frac{\omega_{\mathrm{slip}} L_{\mathrm{m}}}{\omega_{\mathrm{e}} L_{\mathrm{s}}} V_{\mathrm{sd}}^{\mathrm{n}} \\
& V_{\mathrm{rq}}^{\mathrm{n}^{\prime}}=\omega_{\text {slip }} \sigma L_{\mathrm{r}} I_{\mathrm{rq}}^{\mathrm{n}}-\frac{\omega_{\mathrm{slip}} L_{\mathrm{m}}}{\omega_{\mathrm{e}} L_{\mathrm{s}}} V_{\mathrm{sq}}^{\mathrm{n}}
\end{aligned}
$$

where $k_{\mathrm{p} 1}, k_{\mathrm{i} 1}$ and $k_{\mathrm{p} 2}, k_{\mathrm{i} 2}$ are the proportional and integral gains of the positive and negative sequence current controllers respectively.

The rotor control voltage is then transformed to the rotor reference frame as

$$
\boldsymbol{V}_{\mathrm{r}_{-} \alpha \beta}^{*}=\boldsymbol{V}_{\mathrm{r}_{-} \mathrm{dq}}^{\mathrm{p}^{*}} \cdot e^{\mathrm{j}\left(\theta_{\mathrm{s}}-\theta_{\mathrm{r}}\right)}+\boldsymbol{V}_{\mathrm{r}_{-} \mathrm{dq}}^{\mathrm{n}^{*}} \cdot e^{\mathrm{j}\left(-\theta_{\mathrm{s}}-\theta_{\mathrm{r}}\right)} \cdot
$$


Finally PWM patterns for the rotor side converter are generated based on either space vector modulation or sinusoidal PWM method.

\section{SIMULATION STUDIES}

In order to verify the proposed control scheme, simulations for a $2 \mathrm{MW}$ DFIG system shown in Fig. 4 were carried out using Matlab/Simulink. The DFIG system parameters are given in Table I.

Fig. 5 shows the enhanced performance with the proposed scheme compared with the conventional one without taking into account voltage unbalance. In the simulation, $5 \%$ grid voltage unbalance was applied at $0.1 \mathrm{~s}$. The high frequency switching harmonics have been filtered out from the waveforms for clarity. For the conventional control scheme, it can be seen form Fig. 5(A) that the stator current becomes highly unbalanced and the active/reactive power and torque all contain significant $100 \mathrm{~Hz}$ oscillations. For the proposed control scheme, the controller was set to Target 1 during $0.1 \mathrm{~s}-02 \mathrm{~s}$ and switched to Target 2 during 02s-0.3s. As can be seen in Fig. 5(B), the oscillations of the active power and torque have been reduced significantly during $0.1 \mathrm{~s}-02 \mathrm{~s}$ and $02 \mathrm{~s}-0.3 \mathrm{~s}$ respectively.

Fig. 6 shows the DFIG dynamic behaviour when the mechanical torque step-changed between $-0.4 \mathrm{pu}$ and $-1 \mathrm{pu}$ with $5 \%$ voltage unbalance. Target 2 was selected for this case. As can be seen, the positive and negative sequence currents are well controlled, and the electromagnetic torque does not contain
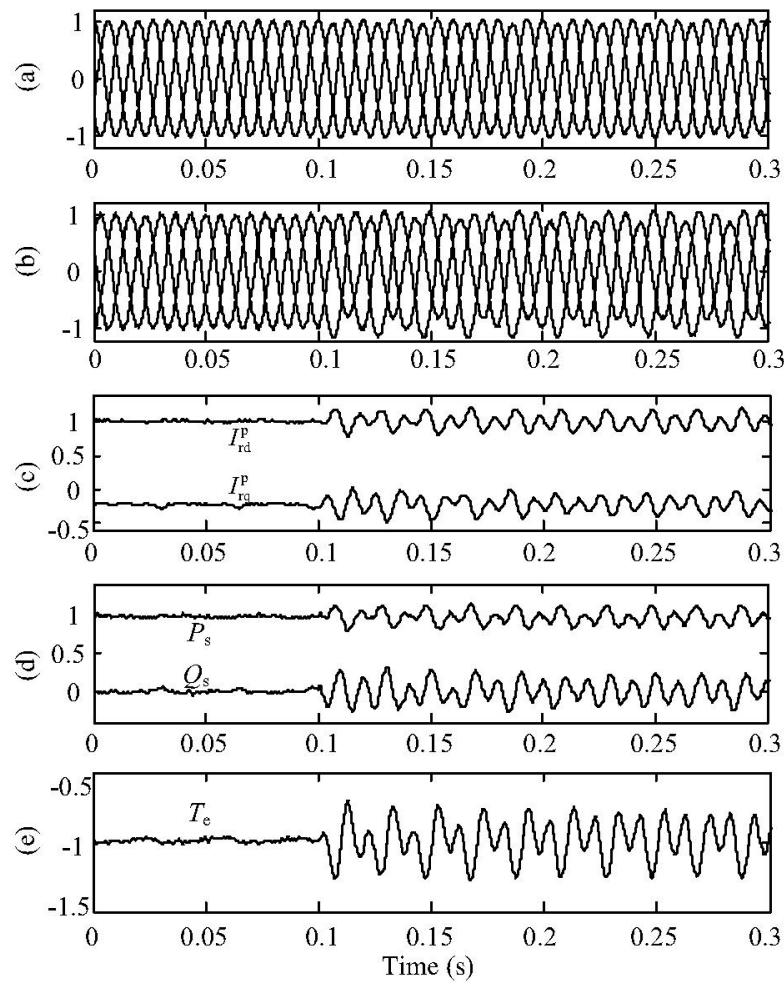

(A) Conventional control scheme

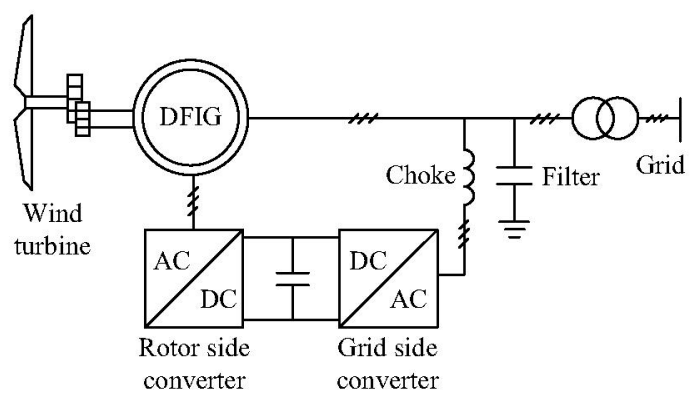

Fig. 4 Schematic diagram of the simulated system

TABLE I

PARAMETERS OF THE SIMULATED DFIG SYSTEM

\begin{tabular}{|c|c|c|}
\hline \multirow{4}{*}{ DFIG } & Rated power & $2 \mathrm{MW}$ \\
\cline { 2 - 3 } & Stator voltage/Frequency & $690 \mathrm{~V} / 50 \mathrm{~Hz}$ \\
\cline { 2 - 3 } & $R_{\mathrm{J}} / R_{\mathrm{I}}$ & $0.0108 \mathrm{pu} / 0.012 \mathrm{pu}$ \\
\cline { 2 - 3 } & $L_{\mathrm{m}}$ & $3.362 \mathrm{pu}$ \\
\cline { 2 - 3 } & $L_{\mathrm{os}} / L_{\mathrm{or}}$ & $0.102 \mathrm{pu} / 0.11 \mathrm{pu}$ \\
\cline { 2 - 3 } & Lumped inertia constant & $1.5 \mathrm{~s}$ \\
\hline DC bus capacitor & $C_{\mathrm{dc}}$ & $10000 \mu \mathrm{F}$ \\
\hline Choke & $L_{\mathrm{g}}$ & $0.25 \mathrm{mH}$ \\
\hline Filter & $R_{\mathrm{f}} / C_{\mathrm{f}}$ & $0.06 \Omega / 1000 \mu \mathrm{F}$ \\
\hline
\end{tabular}

$100 \mathrm{~Hz}$ pulsation.

Fig. 7 shows the simulated results during a remote singlephase fault. The asymmetric fault occurred at $0.2 \mathrm{~s}$ and resulted
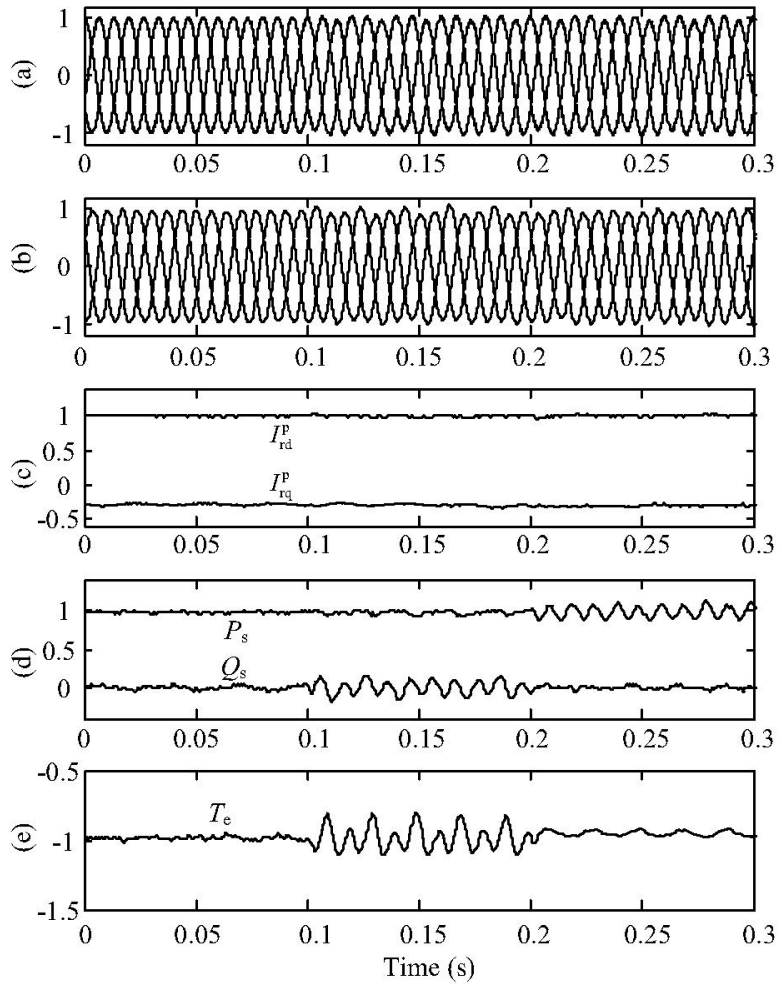

(B) Proposed control scheme

Fig. 5 Simulated results with $5 \%$ unbalanced voltages occurring at $0.1 \mathrm{~s}$, and applying Target 1 in $0.1-0.2 \mathrm{~s}$, Target 2 in $0.2-0.3 \mathrm{~s}$. (a) Stator voltages (pu); (b) Stator currents (pu); (c) Rotor currents in dq reference frame (pu); (d) Active and reactive power of stator side (pu); (e) Electrometric torque (pu) 

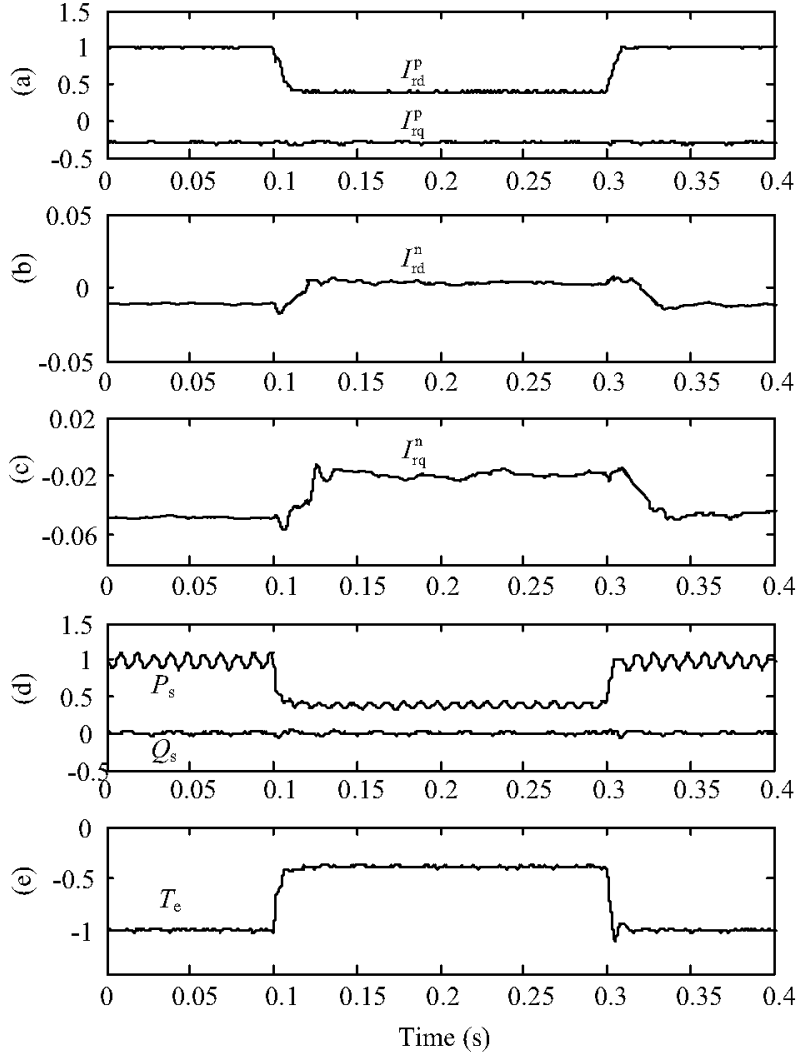

Fig. 6. Simulated results with the variation of input torque by Target 2. (a) Rotor positive sequence currents in dq frame (pu). (b) Rotor negative sequence d-axis current (pu). (c) Rotor negative sequence q-axis current (pu). (d) Active and reactive power (pu). (e) Electromagnetic torque (pu)

in a $10 \%$ negative-sequence voltage and a positive-sequence voltage dip of $7 \%$ on the DFIG terminal (see Fig. 7(a) and 7(b)). The electromagnetic torque behaviour of the proposed control scheme with the controller set Target 2 and that of the conventional control scheme are shown in Fig. 7(c) and 7(d) respectively. As can be seen, the torque ripple is significantly reduced by the proposed scheme.

\section{CONCLUSIONS}

To understand the system operation and to provide enhanced system control for DFIG systems under unbalanced conditions, a dynamic model based on SVO containing the positive and negative sequence components has been developed. Under unbalanced stator supply, torque and power oscillations have been full defined and various control targets to enhance the system operation have been discussed. System control based on a positive sequence controller which controls average active and reactive power, and a negative sequence controller which minimizes torque or power oscillations is designed and validated by simulation. The proposed control strategy can significantly improve the performance and stability of DFIG systems during network unbalance.

\section{REFERENCES}
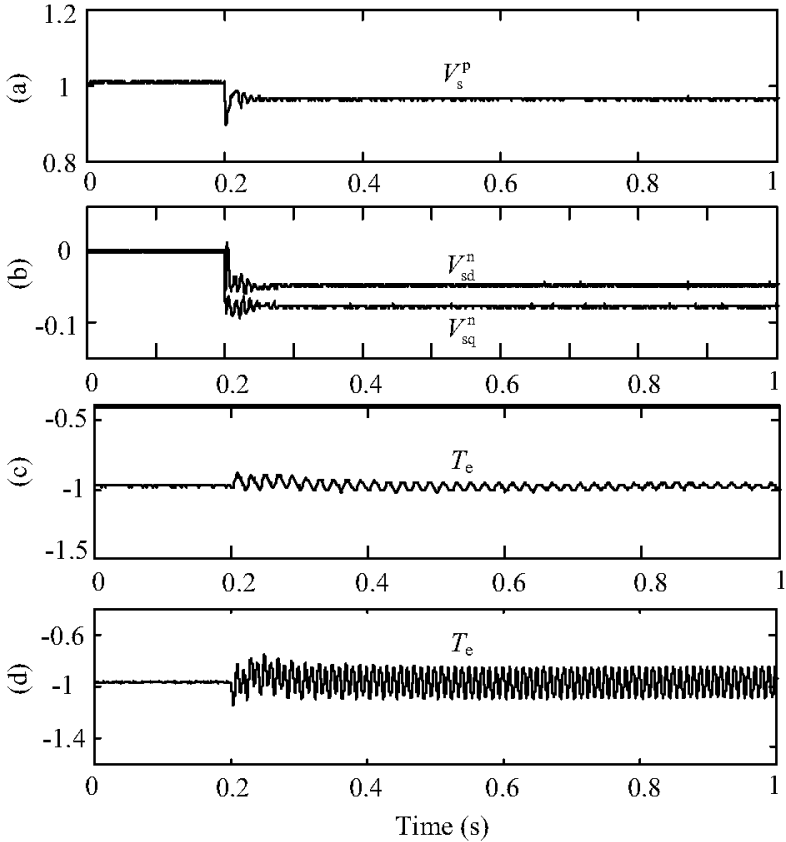

Fig. 7. Simulated results when asymmetric fault occurred at $0.2 \mathrm{~s}$ by Target 2 (a) Stator positive voltage (pu). (b) Stator negative voltages (pu). (c) Electromagnetic torque (pu) by the proposed scheme. (d) Electromagnetic torque (pu) by conventional scheme

[1] F.M. Hughes, O. Anaya-Lara, N. Jenkins, G. Strbac, "Control of DFIGbased wind generation for power network support," IEEE Trans. on Power Systems, vol 20, pp 1958-1966, November 2005.

[2] S. Seman, J. Niiranen, S. Kanerva, A. Arkkio, J. Saitz, "Performance study of a doubly fed wind-power induction generator Under Network Disturbances," IEEE Trans. on Energy Conversion, vol. 21, pp 883-890, December 2006

[3] T. Thiringer, A. Petersson, T. Petru, "Grid disturbance response of wind turbines equipped with induction generator and doubly-fed induction generator," in Proc. IEEE Power Engineering Society General Meeting, vol. 3, pp 13-17, July 2003.

[4] T. Sun, Z. Chen, F. Blaabjerg, "Voltage recovery of grid-connected wind turbines with DFIG after a short-circuit fault, " in Proc. 35th IEEE Power Electronics Specialists Conference, vol. 3, pp 1991-1997, June 2004.

[5] B. I. Nass, T.M. Undeland, and T. Gjengedal, "Methods for reduction of voltage unbalance in. weak grids connected to wind plants," in Proc. IEEE Workshop Wind Power Impacts Power Systems, Oslo, Norway, June 2002.

[6] A. Yazdani, R. Iravani, "A unified dynamic model and control for the voltage-sourced converter under unbalanced grid conditions," IEEE Trans. On Power Delivery, vol. 21, pp 1620-1629, July 2006.

[7] T. Brekken, N. Mohan, T. Undeland, "Control of a doubly-fed induction wind generator under unbalanced grid voltage conditions," in Proc. 2005 European Conference on Power Electronics and Applications, September 2005 .

[8] T. Brekken, N. Mohan, "A novel doubly-fed induction wind generator control scheme for reactive power control and torque pulsation compensation under unbalanced grid voltage conditionsj," in Proc. IEEE 34th Annual Power Electronics Specialist Conference, vol. 2, pp 760-764, June 2003.

[9] L. Xu, Y. Wang, "Dynamic modeling and control of DFIG-based wind turbines under unbalanced network conditions," IEEE Trans. on Power Systems, vol. 22, pp $314-323$, February 2007.

[10] L. Xu; B. R. Andersen, P. Cartwright, "VSC transmission operating under unbalanced AC conditions - analysis and control design," IEEE Trans .on Power Delivery, vol. 20, pp 427 - 434, January 2005. 